\title{
Psychosocial distress among women following a natural disaster in a low- to middle-income country: "healthy mothers, healthy communities" study in Vanuatu
}

\author{
Alysa Pomer ${ }^{1}$ (D) Giavana Buffa ${ }^{2} \cdot$ Marie-Belle Ayoub $^{3}$ - Fasiah Taleo ${ }^{4} \cdot$ J. Hunter Sizemore ${ }^{5}$ - Apisai Tokon ${ }^{4}$. \\ Chim W. Chan ${ }^{6} \cdot$ Akira Kaneko $^{6,7,8} \cdot$ Jimmy Obed $^{4} \cdot$ Jerry laruel $^{4} \cdot$ George Taleo $^{4} \cdot$ Len Tarivonda $^{4}$. \\ Kelsey N. Dancause ${ }^{3}$
}

Received: 2 May 2018 / Accepted: 17 May 2019 / Published online: 5 June 2019

(C) Springer-Verlag GmbH Austria, part of Springer Nature 2019

\begin{abstract}
Natural disasters have major consequences for mental health in low- and middle-income countries. Symptoms are often more pronounced among women. We analyzed patterns and predictors of distress among pregnant and non-pregnant women 3-4 and 15-16 months after a cyclone in Vanuatu, a low- to middle-income country. Distress levels were high among both pregnant and non-pregnant women, although pregnant women showed lower longer-term symptoms. Low dietary diversity predicted greater distress, which could affect women even in villages with little cyclone damage.
\end{abstract}

Keywords Pregnancy $\cdot$ Pacific $\cdot$ PTSD $\cdot$ Developing country $\cdot$ Low- and middle-income countries (LMICs)

\section{Introduction}

Natural disasters affect an increasing number of people globally, especially in low- and middle-income countries (LMICs). Risk for adverse mental health outcomes after natural disasters is often more pronounced among women than men (Norris et al. 2002; Galea et al. 2005; Sohrabizadeh et al. 2016) and might vary based on pregnancy status. Physiological and

Alysa Pomer

alysa.pomer@yale.edu

1 Department of Chronic Disease Epidemiology, Yale School of Public Health, 60 College Street, New Haven, CT 06510, USA

2 Ross University School of Medicine, Portsmouth, Dominica

3 Départment des sciences de l'activité physique, Université du Québec à Montréal, Montréal, QC, Canada

4 Ministry of Health, Port Vila, Vanuatu

5 Peace Corps Vanuatu, Port Vila, Vanuatu

6 Department of Parasitology, Graduate School of Medicine, Osaka City University, Osaka, Japan

7 Island Malaria Group, Department of Microbiology, Tumor and Cell Biology (MTC), Karolinska Institutet, Stockholm, Sweden

8 Institute of Tropical Medicine, Nagasaki University, Nagasaki, Japan emotional responses to stress are attenuated during pregnancy, representing a buffering mechanism for the pregnant woman and fetus (Christian 2012). However, pregnant women face specific burdens, such as disrupted access to obstetric care (ACOG 2010), which could exacerbate distress following natural disasters. Few distress studies following natural disasters in LMICs address variations by pregnancy status.

In March 2015, the lower- to middle-income country of Vanuatu experienced a Category 5 cyclone that left many villages in ruins. As part of a broader study on prenatal stress and infant development, we assessed predictors of maternal distress after the cyclone and compared patterns among pregnant and non-pregnant women.

\section{Materials and methods}

This study was approved by the Human Research Ethics Committee of the University of Quebec in Montreal, and the Vanuatu Minstry of Health.

We created a self-report questionnaire to assess demographics (age, marital status, number of children, years of education), dietary patterns, damage due to the cyclone, and distress due to the cyclone. Adult women on four islands (Aneityum, Efate, Erromango, Tanna) were invited to 
participate. Data were collected 3-4 months after the cyclone, in June-July 2015. On Efate, home of the urban capital, we conducted convenience sampling at local clinics, the women's center, and the open-air market. On the other islands, women were recruited during malariometric surveys in 12 rural communities (Chan et al. 2017). We repeated distress and dietary evaluations on three islands (Aneityum, Efate, Tanna) 1516 months after the cyclone, in June-July 2016.

\section{Instruments}

Surveys were conducted in Bislama, the lingua franca of Vanuatu.

Cyclone damage We assessed damage to women's village, home, and gardens due to the cyclone. Responses were on a 4-point scale from "Good, untouched by cyclone" to "Severe damage, must rebuild." We summed responses across the three categories. Women also reported number of days without adequate food and water following the cyclone.

Distress Distress was assessed using a Bislama adaptation of the Impact of Event Scale-Revised (IES-R) (Weiss and Marmar 1997). Questions were translated to Bislama and reviewed by local speakers. Three questions from each category (intrusive thoughts, hyperarousal, avoidance) were retained in the final questionnaire. Women reported on a scale of 0 ("Not at all") to 4 ("Extremely") the extent to which they experienced symptoms relative to the cyclone. The mean of response values for all 9 items was used for analyses.

Dietary diversity Women completed 24-h dietary recall, and foods were classified into nine groups based on micronutrients using the Women's Dietary Diversity Score (FAO 2010). Dietary diversity was calculated as the number of groups represented in the recall.

\section{Analyses}

We analyzed descriptive statistics, including differences in means among pregnant and non-pregnant women and among islands, using one-way ANOVA.

Predictors of distress We used general linear models to test predictors of distress 3-4 months after the cyclone. Full models included damage to village, home, and garden; days without adequate food; days without adequate water; dietary diversity; island; pregnancy status; and key demographic variables (age, years of education, number of children). We repeated general linear models to test predictors of distress in 2016. Full models included dietary diversity, island, pregnancy status, and key demographic variables.
Prevalence of high distress Although the IES-R suggests cutoffs at which risk of PTSD is likely elevated, we cannot directly apply these to our adapted questionnaire. A cutoff of $\geq$ 37 on the IES-R is suggested as high enough to predict suppressed immune function. This translates to a mean score of 1.7 on our questionnaire, which we used to categorize high distress, to facilitate comparison to other studies.

Statistical significance was defined as $p<0.05$. Analyses were conducted with SPSS 22 (IBM Statistics).

\section{Results}

Surveys were completed by 926 women in 2015 and 1365 in 2016. Table 1 shows descriptive statistics, including comparisons by pregnancy status. Mean scores on the damage questionnaire in 2015, days without adequate food, and days without adequate water differed among islands $(p<0.001)$ (analyses not shown). Despite these differences, dietary diversity scores in 2015 did not differ among islands ( $p=0.438$ ).

Predictors of distress in 2015 Controlling for island $(p=$ 0.067 ), demographic variables (age, $p=0.349$; education, $p=0.556$; number of children, $p=0.581$ ), and days without adequate food $(p=0.602)$ and water $(p=0.316)$, predictors of distress included dietary diversity $(p=0.023)$ and damage to home, village, and garden $(p<0.001)$. Pregnancy status did not predict distress $(p=0.633)$ : adjusting for covariates, mean distress scores were $2.1(95 \% \mathrm{CI}=2.0-2.3)$ among pregnant and $2.2(95 \% \mathrm{CI}=2.1-2.3)$ among non-pregnant women. Table 2 shows parameter estimates for all variables. Replacing the composite damage score with individual scores indicated that damage to the village $(p=0.026)$ and house $(p=0.014)$ predicted distress, whereas damage to the garden did not $(p=0.910)$. Results for other variables were unchanged.

Predictors of distress in 2016 Island $(p=0.099)$, age ( $p=$ $0.554)$, and number of children $(p=0.962)$ did not predict distress, and dietary diversity was no longer a significant predictor of distress in $2016(p=0.066)$. Rather, education was the most robust predictor $(p<0.001)$. Furthermore, pregnancy status predicted distress in $2016(p=0.013)$. Adjusted for covariates, mean distress was lower among pregnant $(1.3,95 \%$ $\mathrm{CI}=1.2-1.4)$ than non-pregnant $(1.5,95 \% \mathrm{CI}=1.4-1.6)$ women.

Prevalence of high distress Based on the cutoff of 1.7, 71.5\% of non-pregnant and $65.9 \%$ of pregnant women $(70.5 \%$ total) had high symptoms of distress in 2015 , and $38.6 \%$ of nonpregnant and $28.9 \%$ of pregnant women (33.7\% total) had high symptoms of distress in 2016. 
Table 1 Descriptive statistics with $p$ values testing differences between pregnant and nonpregnant women

\begin{tabular}{|c|c|c|c|c|c|}
\hline & \multicolumn{2}{|l|}{ Not pregnant } & \multicolumn{2}{|l|}{ Pregnant } & \multirow[t]{2}{*}{$p$ value } \\
\hline & Mean (SD) & $n$ & Mean (SD) & $n$ & \\
\hline \multicolumn{6}{|l|}{2015} \\
\hline Age (years) & $32.9(10.5)$ & 671 & $25.6(5.5)$ & 142 & $<0.001$ \\
\hline Years of school & $6.7(3.7)$ & 642 & $7.3(3.9)$ & 140 & 0.069 \\
\hline Number of children & $3.3(1.9)$ & 669 & $2.0(1.8)$ & 143 & $<0.001$ \\
\hline Dietary diversity & $2.9(1.2)$ & 601 & $3.2(1.1)$ & 130 & 0.032 \\
\hline Distress & $2.2(0.9)$ & 636 & $2.1(0.9)$ & 134 & 0.056 \\
\hline Damage to village, home, garden & $10.9(1.8)$ & 578 & $10.7(1.8)$ & 128 & 0.185 \\
\hline Days without food & $18.8(24.8)$ & 629 & $15.1(22.4)$ & 130 & 0.114 \\
\hline Days without water & $16.0(24.6)$ & 631 & $12.6(23.2)$ & 130 & 0.151 \\
\hline \multicolumn{6}{|l|}{2016} \\
\hline Age (years) & $32.4(11.7)$ & 678 & $25.5(9.0)$ & 646 & $<0.001$ \\
\hline Years of school & $8.2(3.4)$ & 644 & $9.7(3.3)$ & 559 & $<0.001$ \\
\hline Number of children & $2.9(1.8)$ & 685 & $1.3(1.4)$ & 659 & $<0.001$ \\
\hline Dietary diversity & $3.7(1.3)$ & 580 & $4.0(1.3)$ & 572 & 0.004 \\
\hline Distress & $1.5(0.9)$ & 549 & $1.2(0.9)$ & 564 & $<0.001$ \\
\hline
\end{tabular}

\section{Discussion}

Our objective was to assess maternal distress following a natural disaster in Vanuatu, and differences based on pregnancy status. Pregnant women showed slightly lower distress scores than non-pregnant women in 2016, consistent with a stress buffering effect during pregnancy. However, pregnant and non-pregnant women were equally vulnerable to distress 3 4 months after the cyclone, with $65.9 \%$ of pregnant women experiencing symptoms of high distress. This could hold implications for infant development: among pregnant women, greater distress due to the cyclone predicted lower infant birthweight and was a more important predictor than dietary diversity (Pomer et al. 2018). Extra services for pregnant women might need to be prioritized in the early months following a disaster.

As expected, greater damage to the village and home predicted greater distress. Other predictors included lower education and lower dietary diversity. People with greater education might have more resources to aid in recovery, or might be
Table 2 Summary of general linear models (unstandardized coefficients $\beta$, partial $\eta^{2}, p$ values) testing predictors of distress

\begin{tabular}{|c|c|c|c|c|c|c|}
\hline \multirow[t]{2}{*}{ Variable } & \multicolumn{3}{|l|}{2015} & \multicolumn{3}{|l|}{2016} \\
\hline & $\beta$ & $\begin{array}{l}\text { Partial } \\
\eta^{2}\end{array}$ & $p$ value & $\beta$ & $\begin{array}{l}\text { Partial } \\
\eta^{2}\end{array}$ & $p$ value \\
\hline \multicolumn{7}{|l|}{ Island } \\
\hline Efate & 0.164 & 0.002 & 0.304 & 0.119 & 0.001 & 0.269 \\
\hline Tanna & -0.042 & $<0.001$ & 0.810 & 0.308 & 0.005 & 0.033 \\
\hline Erromango* & 0.274 & 0.004 & 0.155 & - & - & - \\
\hline \multicolumn{7}{|l|}{ Aneityum (Ref) } \\
\hline Age (years) & 0.005 & 0.002 & 0.349 & -0.002 & $<0.001$ & 0.554 \\
\hline Years of education & 0.006 & 0.001 & 0.556 & -0.072 & 0.056 & $<0.001$ \\
\hline Number of children & 0.014 & 0.001 & 0.581 & 0.001 & $<0.001$ & 0.962 \\
\hline Pregnant & -0.048 & $<0.001$ & 0.633 & -0.162 & 0.007 & 0.013 \\
\hline Dietary diversity & -0.073 & 0.009 & 0.023 & -0.043 & 0.004 & 0.066 \\
\hline $\begin{array}{l}\text { Hardship (damage to village, home, } \\
\text { garden)* }\end{array}$ & 0.125 & 0.036 & $<0.001$ & - & - & - \\
\hline Days without adequate food* & -0.001 & $<0.001$ & 0.602 & - & - & - \\
\hline Days without adequate water* & 0.002 & 0.002 & 0.316 & - & - & - \\
\hline
\end{tabular}

Italicized entries indicate statistically significant predictors of distress

*Assessed only in 2015 
more likely to seek mental health services. Limited dietary diversity predicted distress independent of cyclone damage, highlighting broader effects of the disaster on women's psychological health even among those with little direct exposure. This underscores the importance of broadening interventions across the region, rather than only in severely damaged communities.

Despite limited healthcare infrastructure, the importance of mental health interventions following disasters is increasingly recognized in LMICs. In Vanuatu, mental health gained increased public attention following the cyclone, and more local health professionals have participated in mental health training workshops. This contributed to a first-of-its-kind response in Vanuatu in 2017, when the entire population of one island was evacuated due to volcanic activity. A small team was sent to provide professionally led group discussions and individual psychological first aid in some displacement camps. Our studies showed that these services were used along with traditional support systems such as local healers, and that women who did not have access to these support networks had higher distress (Zahlawi et al. 2019). Training local non-specialists such as community healthcare workers to provide mental health services is a promising strategy for increasing mental health capacity in LMICs (Kakuma et al. 2011). Local community workers might be well-positioned to adapt interventions to the cultural context, which helps to lay foundations for long-term continuation.

Study limitations include the convenience sample and selfreport instruments. Although we sampled in the same communities in 2015 and 2016, the samples do not include the exact same participants and we cannot assess longitudinal changes in distress. Furthermore, our adaptation of the IES$\mathrm{R}$ cannot be directly compared to the original, and our figures on prevalence of high distress must be interpreted cautiously.

As the number and severity of natural disasters increases in LMICs, the need for tailored and sustainable mental health interventions will continue to grow. Our results highlight the importance of broadening interventions across the larger region, rather than only among those from severely damaged communities. Pregnant women are equally vulnerable to shorter-term distress symptoms, highlighting the need for services in the early months following the disaster. Furthermore, although distress symptoms decrease with time, a substantial part of the population (including pregnant, but especially nonpregnant women) experiences longer-term symptoms. Ultimately, small-scale interventions following disasters might lay the foundation for development of broader mental health infrastructure in LMICs, which represents a major public health priority (Epping-Jordan et al. 2015).

Acknowledgments The "Healthy Mothers, Healthy Communities" project grew out of our collaborations with the Health Transition in Vanuatu research team, led by Ralph Garruto and J. Koji Lum (Binghamton
University), and the Stress in Pregnancy International Research Alliance, led by Suzanne King (McGill University). We are deeply appreciative to the local malaria team, who continue to allow us to work alongside them in the outer islands, including Harry Iata, James Yaviong, Morris Kalkoa, and Sam Yamar. We are grateful to the women in the Vila Central Market who helped distribute questionnaires; Christiane Damassing for her assistance with recruitment; Alek Buffa, who helped with data collection; and those women in each of the villages who completed the survey themselves, then encouraged and aided their peers to complete the survey as well. Thanks to Harold Neel for his continued local support; without which, this study would not have been possible.

Funding Supported by funds from the Wenner-Gren Foundation for Anthropological Research, the Natural Hazards Center, and the Faculté des sciences of the Université du Québec à Montréal. Kelsey Dancause was supported by a salary award from the Fonds de recherche du Québec - Santé while working on this project.

\section{Compliance with ethical standards}

Ethical approval All procedures performed in studies involving human participants were in accordance with the ethical standards of the institutional and/or national research committees and with the 1964 Helsinki declaration and its later amendments or comparable ethical standards.

Informed consent Informed consent was obtained from all individual participants included in the study.

Conflict of interest The authors declare that they have no conflicts of interest.

\section{References}

American College of Obstetricians and Gynecologists (ACOG) (2010) ACOG Committee Opinion No. 457: preparing for disasters: perspectives on women. Obstet Gynecol 115(6):1339-1342

Chan CW, Iata H, Yaviong J, Kalkoa M, Yamar S, Taleo G, Isozumi R, Fukui M, Aoyama F, Pomer A, Dancause KN, Kaneko A (2017) Surveillance for malaria outbreak on malaria-eliminating islands in Tafea Province, Vanuatu after Tropical Cyclone Pam in 2015. Epidemiol Infect 145(1):41-45

Christian LM (2012) Physiological reactivity to psychological stress in human pregnancy: current knowledge and future directions. Prog Neurobiol 99(2):106-116

Epping-Jordan JE, van Ommeren M, Ashour HN, Maramis A, Marini A, Mohanraj A, Noori A, Rizwan H, Saeed K, Silove D, Suveendran T, Urbina L, Ventevogel P, Saxena S (2015) Beyond the crisis: building back better mental health care in 10 emergency-affected areas using a longer-term perspective. Int J Ment Heal Syst 9:15

Food and Agriculture Organization (FAO) (2010) Guidelines for measuring household and individual dietary diversity. Rome, Food and Agriculture Organization of the United Nations. http://www.fao. org/3/a-i1983e.pdf. Accessed 2 May 2018

Galea S, Nandi A, Vlahov D (2005) The epidemiology of post-traumatic stress disorder after disasters. Epidemiol Rev 27(1):78-91

Kakuma R, Minas H, van Ginneken N, Dal Poz MR, Desiraju K, Morris JE, Saxena S, Scheffler RM (2011) Human resources for mental health care: current situation and strategies for action. Lancet 378(9803):1654-1663

Norris FH, Friedman FJ, Watson PJ (2002) 60,000 disaster victims speak: part I and II. Psychiatry 65(3):207-260 
Pomer A, Buffa G, Taleo F, Sizemore JH, Tokon A, Taleo G, Tarivonda L, Chan CW, Kaneko A, Dancause KN (2018) Relationships between psychosocial distress and diet during pregnancy and infant birthweight in a lower-middle income country: "healthy mothers, healthy communities" study in Vanuatu. Ann Hum Biol 45(3): 220-228

Sohrabizadeh S, Tourani Ph DS, Khankeh HR (2016) Women and health consequences of natural disasters: challenge or opportunity? Women Health 56(8):977-993

Weiss DS, Marmar CR (1997) The impact of event scale - revised. Guilford, New York
Zahlawi T, Roome AB, Chan CW, Campbell JJ, Tosiro B, Malanga M, Tagaro M, Obed J, Iaruel J, Taleo G, Tarivonda L, Olszowy KM, Dancause KN (2019) Psychosocial support during displacement due to a natural disaster: relationships with distress in a lower-middle income country. Int Health. https://doi.org/10.1093/inthealth/ihy099

Publisher's note Springer Nature remains neutral with regard to jurisdictional claims in published maps and institutional affiliations. 